\title{
Optical millimeter wave generation utilizing stimulated brillouin scattering for radio over fiber system
}

\author{
M.M.H Husaini, C.B.M Rashidi, SN Azemi, S.A Aljunid, M.S Anuar \\ Advanced Communication Engineering, Centre of Excellence, School of Computer and Communication Engineering, \\ Universiti Malaysia Perlis (UNIMAP), Perlis, Malaysia
}

\begin{abstract}
Article Info
Article history:

Received Oct 25, 2018

Revised Dec 16, 2018

Accepted Dec 29, 2018

\section{Keywords:}

Bit Error Rate (BER)

External modulation

Milimeter Wave

Radio over Fiber (RoF)

ABSTRACT

Optical millimeter-wave signal generation in Radio over Fiber (RoF) based on external modulators using different modulation schemes such as SingleSideband (SSB), Double-Sideband (DSB) are experimentally compared. The major fallout for external modulation scheme system performance to downgrade is due to dispersion and the fading effect in optical signal generation while generating high Bit Error Rate (BER) when involving long distance delivery. In this work, we proposed mm-wave signal utilize Stimulated Brillouin Scattering (SBS) scheme which indicates the best performance amongst the scheme based on simulation that it achieves to propagate longest of distance over fiber, highest receiver sensitivity, the lowest BER and coherence of Mach-Zehnder Modulator (MZM) extinction ratio. Mm-wave signal of $40 \mathrm{Ghz}$ and $10 \mathrm{~Gb} / \mathrm{s}$ data rate was generated with the plotting of eye diagram.
\end{abstract}

Stimulated Brillouin Scattering
Copyright $@ 2019$ Institute of Advanced Engineering and Science. All rights reserved.

\section{Corresponding Author:}

Muhammad Haziq Husaini Bin Mazelan,

School of Computer and Communication (SCCE),

c/o School of Manufacturing Engineering Complex,

Universiti Malaysia Perlis (UniMAP),

First floor, Pauh Putra Campus, 02600 Arau, Perlis.

Email: haziqhusaini@studentmail.unimap.edu.my

\section{INTRODUCTION}

The controversial matter of spectral congestion at low frequency ranges will survive by the invention of Millimeter Wave (mm-wave) signal generation. Thus, acknowledge the Radio-Over-Fiber (RoF) performance system since it has low attenuation, large bandwidth and could resist to Electormagnetic Interference (EMI) which these system can be used in various types of application [1-2]. RoF is a transmission link which light propagates by Radio Frequency (RF) and transmitted by optical fiber link where optical mm-wave generation and all-optical up-conversion are the fundamental approach in RoF.

The typical schematic of RoF link is shown in Figure 1 which laser light is modulated by radio signal and transported over an optical fiber medium. Considering the radio-frequency carrier signal as an analog signal, which make up the laser modulation an analog signal too. If frequency conversion is utilized, the modulation may occur at radio signal frequency or any intermediate frequency [3-4].

A bidirectional interface containing the analog laser transmitter, photodiode receiver located at a base station or remote antenna unit, paired with an analog laser transmitter and photodiode receiver located at a radio processing unit are the main configuration content of analog fiber optic link. Remote antenna unit is connected to central processing location by one or more optical fiber [5].

Various studies had been widely reported over the past few years regarding the transmission of optical mm-wave for future generation such as direct modulation [6], external modulation [7] and optical heterodyne [8]. However, out of all, the simplest scheme is concluded to be direct modulation despite lacking of few factors such as low frequency response and are limited by the laser frequency chirp. In contrasts, the 
optical heterodyne technique can produce high frequency response, unfortunately the signal quality for this scheme depends on the coherence of two laser lightwaves. As demonstrated by researchers, the most popular method that practically been used is external modulation [9]. There are two types of external modulators: Electro Absorption Modulators (EOM) and Mach-Zehnder Modulator (MZM). The most commonly used external modulator is MZM.

Single-Sideband (SSB), Double-Sideband (DSB) and Optical Carrier Suppression (OCS) are commonly used for signal generation in the external modulation scheme. Upon extending the delivery distance for the optical millimeter wave, SSB modulation is superior to DSB due to its reduced effects in suffering dispersion in a single-mode fiber (SMF), but the receiver reactivity for SSB is comparatively low due to the huge dc component at the central wavelength. A Fiber Bragg Grating (FBG) are used to conceal the dc component in order to improve the receiver sensitivity [10].

However, the FBG is hypersensitive to temperature, which will need a convoluted control system to maintain its operation besides most power will be lost due to FBG reflection. When a single-mode laser is transmitted over a $\mathrm{LiNbO}_{3}$ modulator (LN-MOD), a dual-mode lightwave can be generated based on optical carrier suppression (OCS) modulation. The dual-mode lightwave are then separated before another one mode is used to carry the optical millimeter wave by using an optical filtering technique. The configuration of this optical millimeter-wave source is complex and unstable [11] for long-haul application since the FBG filters and other optical components are used.

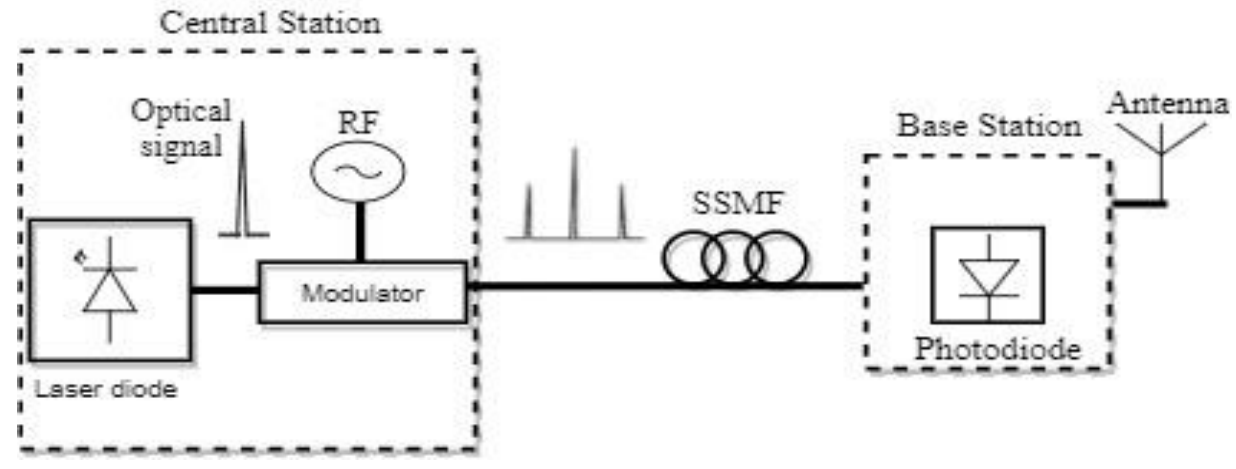

Figure 1. A generic schematic diagram of fiber-radio link for mm-wave signal generation

In this paper, an alternative technique is proposed which is Stimulated Brillouin Scattering (SBS) to overcome and enhance above mentioned problem. SBS is the main nonlinear effect in optical light wave systems for acute linewidth optical sources and has been widely analyzed in depth for optical fiber communications [12]. In this paper, we experimentally demonstrated SBS technique and compare the performances of the optical mm-waves signal generation based on external modulation scheme using SSB and DSB.

\section{COMPARISON OF OPTICAL MM-WAVE SIGNAL METHOD}

Figure 2 shows the schematic optical generation of mm-wave using SBS technique. Within the optical modulator part, it consist of Continuous Wave $(\mathrm{CW})$ laser at $1550 \mathrm{~nm}$ with an intensity-modulated via MZM driven by electrical sine generator of, $F_{r f}=40 \mathrm{GHz}$. A dual series of MZM components are used where MZM 1 modulates the optical root source and MZM 2 is modulated via pseudo-random bit sequence (PRBS) with NRZ electrical signal to produce baseband data signal. The voltage that is linked on MZM is adequately high so that the laser wave will regulated nonlinearly with the given frequency of the electrical generator.

The few optical sidebands deserted by frequency of electrical generator from the optical carrier are produced by MZM non linearity. The distance of transmission are linked up to into $100-\mathrm{km}$ long of Single Mode Fiber (SMF). With the use of an optical amplifier, the optical signal is then amplified before being launched into the optical fiber loop. The amplifier which act as control power of signal wave generates sidebands by nonlinear modulation from CW laser will be amplified by SBS in an optical fiber, whereas the rest will be attenuated due to the process of natural attenuation in the fiber.

The combined output signal of two pump sources driven by an optical circulator that propagates modulated signals through opposite direction are created due to the SBS effects gain in the SMF. The

Optical Millimeter Wave Generation utilizing Stimulated Brillouin Scattering for... (M.M.H Husaini) 
wavelength of each pump laser is set to $980 \mathrm{~nm}$. Therefore, the photodiode detected the outcome of generated mm-wave output. Inset (i) and (ii) shows the optical spectrum when the signal is propagated and ideal eye diagram of SBS scheme.

For DSB modulation, the frequency of the driven RF signal is $40 \mathrm{GHz}$, the generated millimeter wave can take up over $80-\mathrm{GHz}$ bandwidth since it has two sidebands. Due to having different velocities in SMF, the RF power at $40 \mathrm{GHz}$ will disappear after transmitting over a certain length of SMF. In addition, for SSB modulation a dual arm MZM is employed to achieve the modulation where two electrical RF signals drive the dual-arm MZM have a phase shift of $\frac{\pi}{2}$ and the DC bias is at $0.5 V_{\pi}$. The generated optical millimeter wave covers only 40-GHz.

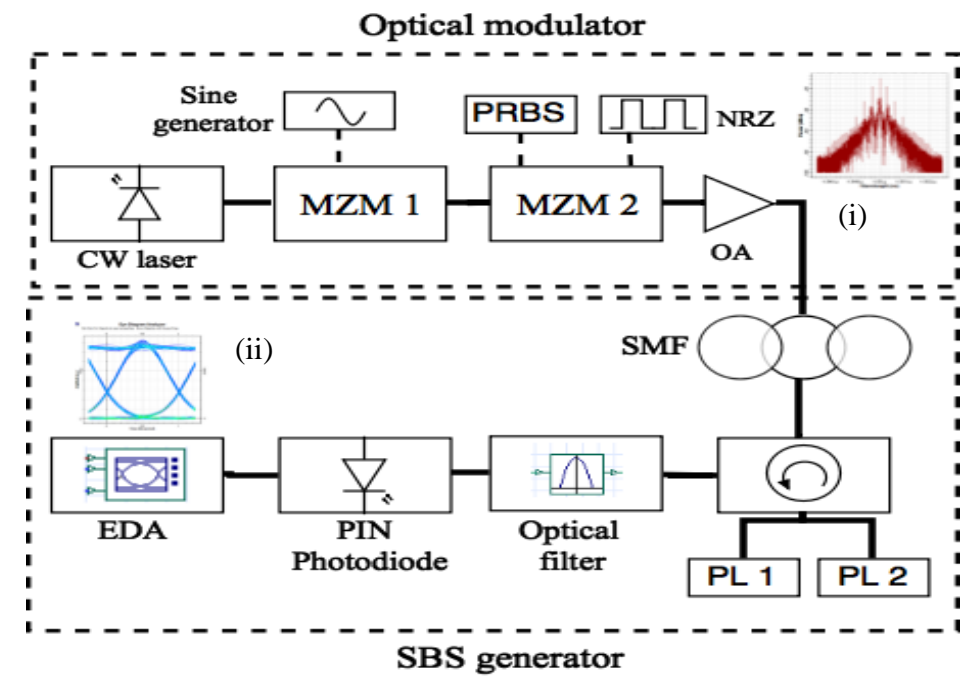

Figure 2. Block diagram of optical mm-wave for SBS technique. (CW: Continuous Wave, OA: Optical Amplifier, SMF: Single Mode Fiber, PL1 \& PL2: Pump Laser, EDA: Eye Diagram Analyzer) inset (i) Optical spectrum (ii) Eye diagram

\section{PERFORMANCE EVALUATION}

In order to design the system, it is crucial to comply the system design with the BER requirement of the network. Generally, the Q-factor provides a qualitative description of the receiver outcomes because it is a function of the Signal to Noise Ratio (SNR). The lowest SNR requisite to get a specific BER for a given signal are suggested by Q-factor. Mathematically, Q-factor of an optical signal is represented by:

$$
Q=\frac{I_{1}-I_{0}}{\sigma_{1}-\sigma_{0}}
$$

This is where $I_{1}$ the value of the 1-bit current, $I_{0}$ is the value of the 0-bit current, while $\sigma_{1}$ is the standard deviation of the 1-bit current, and $\sigma_{0}$ is the standard deviation of the 0-bit current. Therefore, the connection of Q-factor to BER is presented in (2):

$$
\mathrm{BER}=\frac{1}{2} \operatorname{erfc}\left(\frac{Q}{2}\right)
$$

\section{RESULT AND DISCUSSION}

The parameter setup value as shown in the following table are the generated optical mm-wave value for SSB, DSB and SBS scheme performance which validate by simulation using OptiSystem software. In order to demonstrate the propose scheme, the system is evaluated by referring to BER curve, received power, Q-factor and eye diagram. Typical parameters for simulation as shown in Table 1. 
Table 1. Typical parameters for simulation

\begin{tabular}{cc}
\hline Parameters & Value \\
\hline Wavelength & $1550 \mathrm{~nm}$ \\
Input power & $0 \mathrm{dBm}$ \\
Bit rate & $10 \mathrm{Gbps}$ \\
Fiber length & $80 \mathrm{~km}$ \\
Extinction ratio & $30 \mathrm{db}$ \\
Attenuation & $0.2 \mathrm{db} / \mathrm{km}$ \\
Dispersion & $16.75 \mathrm{ps} / \mathrm{nm} / \mathrm{km}$ \\
\hline
\end{tabular}

Figure 3 shows the optical mm-wave signal utilizes DSB, SSB and SBS scheme has been configured by varying fiber length over BER. The graph depicts the comparison of all three different optical mm-wave performances with fiber length versus BER rate where it clearly indicates BER value increases as fiber distance increase. Based on the analysis made, it clearly stated that optical mm-wave signal by DSB method are only capable to generate signal as far as $20-\mathrm{km}$, where this scheme does not compliance in large-scale communication networks.

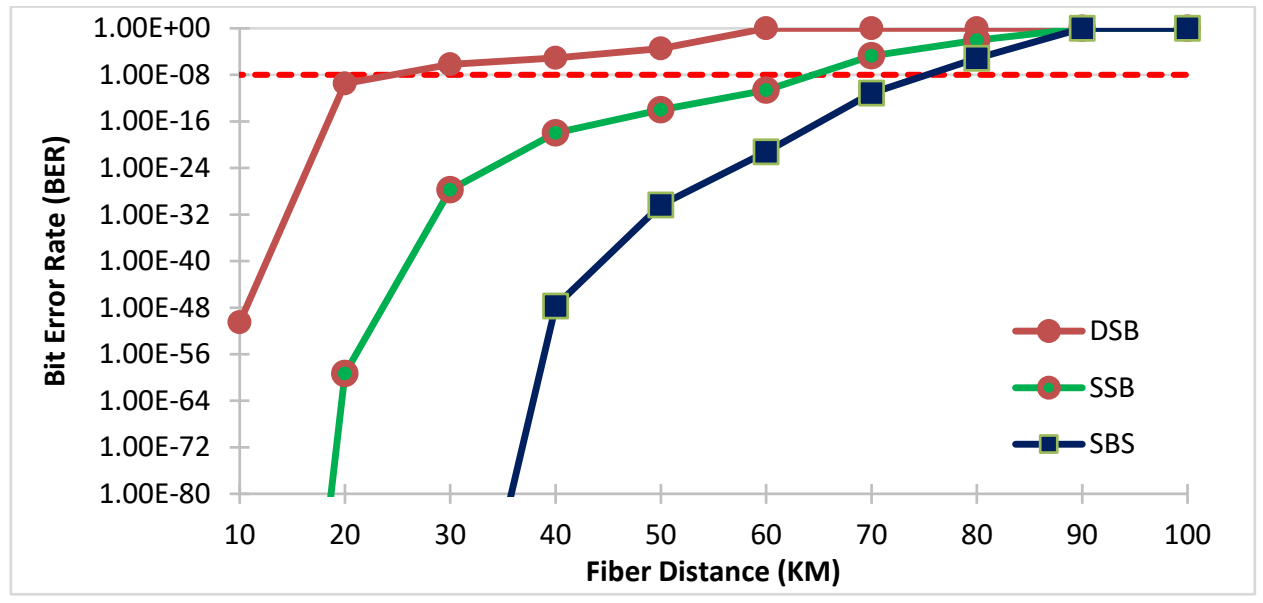

Figure 1. BER versus fiber distance for SSB, DSB, SBS scheme

Meanwhile, the SSB and SBS method can generate signal up to 60-km and 70-km respectively before an error-floor of $10^{-9}$ appeared after it is transmitted over $80-\mathrm{km}$ with bit rate simulation function at $10 \mathrm{Gbps}$, attenuation of $0.2 \mathrm{~dB} / \mathrm{km}$ and dispersion of $16.75 \mathrm{ps} / \mathrm{nm} / \mathrm{km}$ is activated. Therefore, it can be said that SBS scheme improved the system by $16 \%$ system efficiency compared to SSB. As for the fiber length difference at $50-\mathrm{km}$, SSB received higher BER rate which is $10^{-18}$ compared to SBS scheme which is $10^{-28}$. This reinforces the fact that SBS system can generate better signal output in optical mm-wave. However, performance of the system degraded over longer distance due to attenuation and dispersion be found. Thus, high BER can defect the system due to signal losses.

For a better identification, the optical power received is measured on modulated scheme at $30-\mathrm{km}$ transmission. Figure 4 shows the measured BER curve versus received power. The simulation varying input power from $-10 \mathrm{dBm}$ to $0 \mathrm{dBm}$ are conducted. As analyzed from the result shown, the average received optical power of SBS, SSB and DSB system measurement is $-50 \mathrm{dBm},-53.7 \mathrm{dBm}$ and $-57.6 \mathrm{dBm}$ respectively at $20-\mathrm{km}$ transmission. At that particular distance, the lowest BER value for SBS technique is at $10^{-39}$ while SSB scheme manage to achieve the rate of $10^{-32}$ when the input power is set to $0 \mathrm{dBm}$. By imposing $0 \mathrm{dBm}$ as an ideal power launch, it will produce an optimum and sufficient power to generate the signal.

Thus, its proven that SBS scheme can accommodate low received power besides maintaining a low BER measurement. Therefore, the input power parameter is vital as to improve the system performance as demonstrated. When input signal decreases, high BER will be produced. The inset of Figure 4 shows the eye pattern of the received power for SBS technique. 


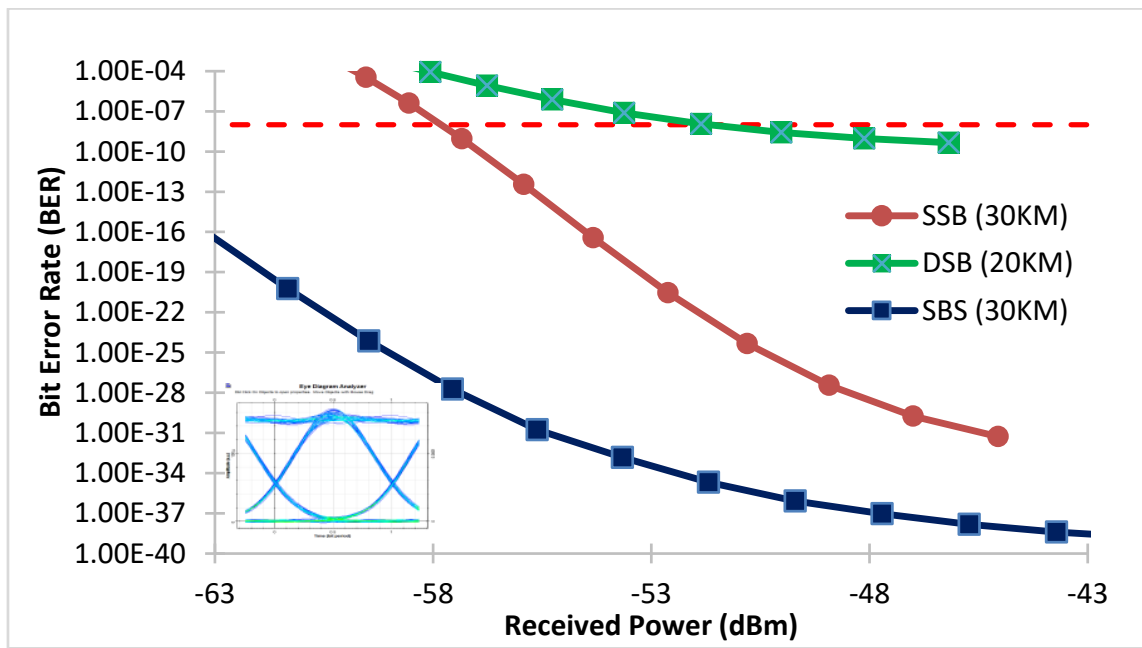

Figure 4. BER versus received power for SSB-30km, DSB-20km and SBS-30km fiber length.(inset shows eye pattern for SBS at $30-\mathrm{km}$ )

Figure 5 depicts the curve of Q-factor against MZM extinction ratio by adjusting the value from 9 $\mathrm{dB}$ to $30 \mathrm{~dB}$ at $25-\mathrm{km}$ transmission. The MZM extinction ratio plays an important role by ensuring the result of output receiver is highest, and in its best quality. According to the graph, SBS method has a higher Qfactor as opposed to SSB and DSB respectively.

Q-factor and BER are interconnected with each other where its Q-factor of 6 corresponding to $\mathrm{BER}=10^{-9}$. For the system performance Q-factor of 6 at $25-\mathrm{km}$, only DSB scheme are reach the lowest system limit at the extinction ratio rate of $15 \mathrm{~dB}$ while SSB and SBS still produced good valuable quality signal at the length used. Thus, it is concluded that SSB and SBS scheme has more flexibility also capabilities to maintained and sustained compared to DSB.

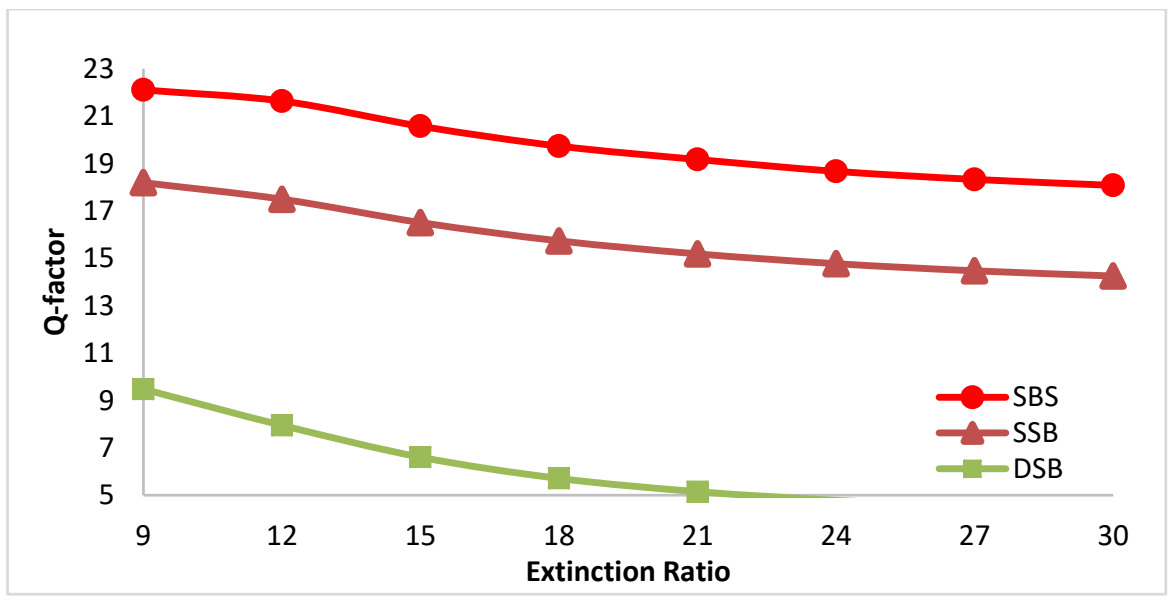

Figure 5. Q-factor versus MZM's extinction ratio for SSB, DSB, and SBS at 25-km transmission distance

As for DSB, SSB and SBS scheme, the demodulation results analysis of eye diagram after transmitting the signal over the length are shown in Figure 6. From the result, it is shown that the eye pattern of DSB scheme when transmitted at $20-\mathrm{km}$ is getting folded which head to large dispersion and performance degraded over ample number of distance. Besides that, the height of eye diagram for SSB and SBS scheme gradually lessened when travelling at $60-\mathrm{km}$ and $70-\mathrm{km}$ respectively. Therefore, the execution of optical mmwave signal generation can be wrapped up by referring to the eye diagram as a gauge or sign of system performance. 


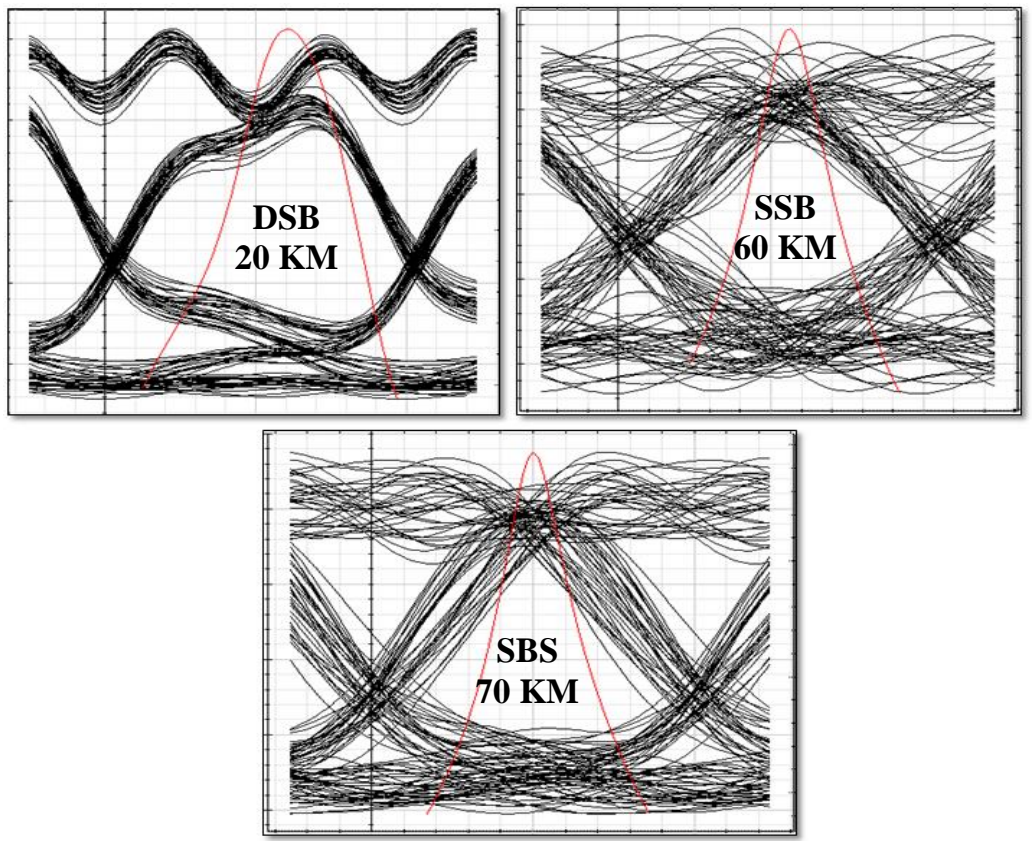

Figure 6. Optical mm-wave eye diagram signal after transmiting over fiber result: (a) DSB-20km (b) SSB$60 \mathrm{~km}(\mathrm{c})$ SBS-70km

\section{CONCLUSION}

In this paper, we have evaluated and demonstrated the optical mm-wave signal generation based on SSB, DSB and SBS scheme. The analysis proves that SBS scheme for RoF system lead the performance comparison of highest received power, minimize BER and longest transmission over distance. The simulation design visualized that the method successfully received average power of $-50 \mathrm{dBm}$ at $20-\mathrm{km}$, transmitted the signal over 70-km fiber length which achieve to improve the system by $10 \%$ over SSB scheme. Thus, it is strongly proven that the proposed method is essential for wide scale communication system. Finally, it is recommended in future work research paper to generate the modulation design widely with different data rate parameter.

\section{REFERENCES}

[1] C. B. M. Rashidi, S. A. Aljunid, F. Ghani, H. A. Fadhil, M. S. Anuar, and A. R. Arief, "Cardinality enrichment of flexible cross correlation (FCC) code for SAC-OCDMA system by alleviation interference scheme (AIS)," Optik (Stuttg)., vol. 125, no. 17, pp. 4889-4894, 2014.

[2] C. B. M. Rashidi, S. A. Aljunid, F. Ghani, M. S. Anuar, and H. A. Fadhil, "Code length optimization using Flexible Cross Correlation (FCC) code in OCDMA networks," ICP 2012 - 3rd Int. Conf. Photonics 2012, Proc., no. October, pp. 355-359, 2012.

[3] C. B. M. Rashidi, S. A. Aljunid, M. S. Anuar, H. A. Fadhil, and F. Ghani, "Performance analysis of a new class of Ccode with flexible cross correlation for SAC-OCDMA system," J. Theor. Appl. Inf. Technol., vol. 61, no. 1, pp. 155-159, 2014.

[4] C. B. M. Rashidi, S. A. Aljunid, F. Ghani, H. A. Fadhil, and M. S. Anuar, "Cardinality enhancement using Flexible Cross Correlation (FCC) code for Spectral Amplitude Coding Optical Code Division Multiple Access systems," $J$. Appl. Sci. Res., vol. 8, no. 12, pp. 5614-5626, 2012.

[5] V. A. Thomas, M. El-Hajjar, and L. Hanzo, "Performance Improvement and Cost Reduction Techniques for Radio Over Fiber Communications," IEEE Commun. Surv. Tutorials, vol. 17, no. 2, pp. 627-670, 2015.

[6] G. D. F. B. Lasers et al., "Optical Microwave / Millimeter-Wave Links Using Direct Modulation of Two-Section," vol. 17 , no. 8, pp. 1734-1736, 2005.

[7] T. Kuri, K. Kitayama, and S. Member, "Fiber-Optic Millimeter-Wave Downlink System," vol. 17, no. 5, pp. 799806, 1999.

[8] T. Kuri and K. I. Kitayama, "Optical heterodyne detection technique for densely multiplexed millimeter-wave-band radio-on-fiber systems," J. Light. Technol., vol. 21, no. 12, pp. 3167-3179, 2003.

[9] C. Jiang, X. Chen, and Z. Liu, "Effect of Modulation Index of Mach-Zehnder Modulator on Single Sideband Radio over Fiber System," Proc. 2012 2nd Int. Conf. Comput. Inf. Appl. (ICCIA 2012), no. Iccia, pp. 574-577, 2012.

[10] T. Geernaert et al., "Transmission improvement in fiber wireless links using fiber Bragg gratings," IEEE Photonics Technol. Lett., vol. 17, no. 1, pp. 190-192, 2005. 
[11] S. Yaakob et al., "Characterisation of DSB-OCS technique for 40GHz radio over fibre system," APCC 2012 - 18th Asia-Pacific Conf. Commun. "Green Smart Commun. IT Innov., no. 1, pp. 612-616, 2012.

[12] R. Chi, L. Li, X. Li, and X. Sun, "STIMULATED BRILLOUIN SCATTERING SUPPRESSED EDFA IN A LONG-HAUL OPTICAL FIBER LINK SYSTEM,” pp. 14-16, 2015.

\section{BIOGRAPHIES OF AUTHORS}

Muhammad Haziq Husaini Bin Mazelan received the B.Eng. in Communication
Engineering from University of Malaysia Perlis (UniMAP), Malaysia. He is currently
completing the M.Sc. degree in Communication Enginering at University of Malaysia
Perlis (UniMAP), Malaysia. His research interest include radio over fiber,millimeter
wave, nonlinear optics and optical communication system.

